\title{
Certification and uncertainty evaluation of flavonoids certified reference materials
}

\author{
Can Quan*, Huanfang Yao, Caixia Hou
}

Division of Chemistry, National Institute of Metrology, Beijing 100013, China;

*Corresponding Author: superfluidcan@hotmail.com

Received August 2013

\begin{abstract}
Flavonoids are the most widely used in the field of the food, medicine, and cosmetic due to their pharmacological functions and biological activities, such as anti-tumor, anti-inflammatory and antioxidation. This work described the assignment of purity values to 3 flavonoids certified reference materials (CRMs) including baicalein, 5,7-dihydoxy flavones and naringenine which were developed in this study according to the ISO Guides 34 and 35 . The qualitative analysis was performed by liquid chromatography-mass spectrometry (LC-MS) and infrared spectroscopy (IR). The CRMs' purity values were assigned based on the weighted average of quantitative nuclear magnetic resonance method and mass balance approach with high resolution liquid chromatography-VWD. All the three CRMs with following value mass fractions: baicalein at a certified purity $P \pm U(k=2)$ of $98.8 \% \pm 0.8 \%$; 5,7-dihydoxy flavones of $99.1 \% \pm 0.7 \%$ and naringenine of $99.5 \% \pm 1.0 \%$ respectively. The homogeneity of the CRMs was determined by an in-house validated liquid chromatographic method. Potential degradation during storage was also investigated and a shelf-life based on this value was established.
\end{abstract}

Keywords: Flavonoids; Certified Reference Material (CRM); Quantitative Nuclear Magnetic Resonance (QNMR); Mass balance approach; Uncertainty

\section{INTRODUCTION}

A certified reference material (CRM) is a material or substance whose one or more property values are sufficiently homogeneous, stable, and well established to be used for the calibration of an apparatus, the assessment of a measurement method, or for assigning values to ma- terials [1].

Flavonoids are increasingly in demand in the field of the food, medicine, and cosmetic [2] due to their pharmacological functions and biological activities, such as anti-tumor, anti-inflammatory and antioxidation [3-5]. The qualities of flavonoids products will have analytical challenges. The flavonoids CRMs have been determined by at least two independent analytical methods. The flavonoids CRMs could be used to ensure the accuracy, traceability and comparability of the related products test results.

This paper describes the development of three flavonoids CRMs including baicalein, 5,7-dihydoxy flavones and naringenine. Qualitative analysis was carried out by infrared spectrometry (IR) and liquid chromatographymass spectrometry (LC-MS). The moisture content and inorganic elements content were determined by Karl Fischer titration, inductively coupled plasma mass spectrometry (ICP-MS), respectively. Homogeneity study was evaluated by F-test and $t$-test both for between vials and within vial. The uncertainties of CRMs were evaluated extensively.

\section{EXPERIMENTAL}

\subsection{Materials}

The chemical structure of flavonoid compounds studied including baicalein, 5,7-dihydoxy flavones and naringenine were shown in Figure 1, all were purchased from Sigma-Aldrich (USA). Benzoic acid certified reference material $(99.9 \% \pm 0.1 \%$ purity) used as QNMR internal standard was obtained from NIST, China.

HPLC grade Acetonitrile and methanol were bought from Merck (HPLC, Germany). Dimethyl sulfoxide- $\mathrm{d}_{6}$ solvent was purchased from Cambridge Laboratoties (USA). Distilled deionized water $(18.2 \mathrm{M} \Omega \cdot \mathrm{cm})$ was obtained with Milli-Q water system and filtered through $0.22 \mu \mathrm{m}$ membrane.

\subsection{Instrument}

Infrared spectra were collected with FT-IR spectrometer 


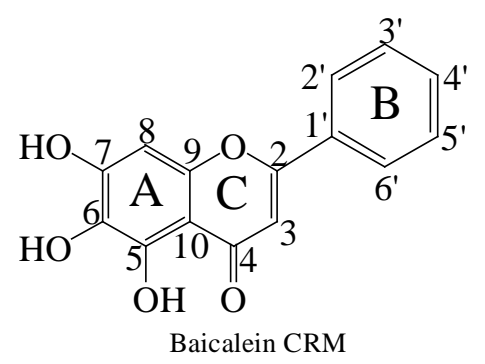

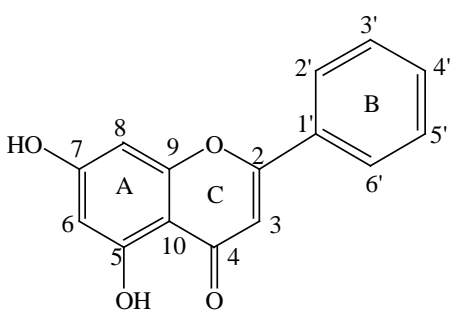

5,7-dihydoxy flavones CRM

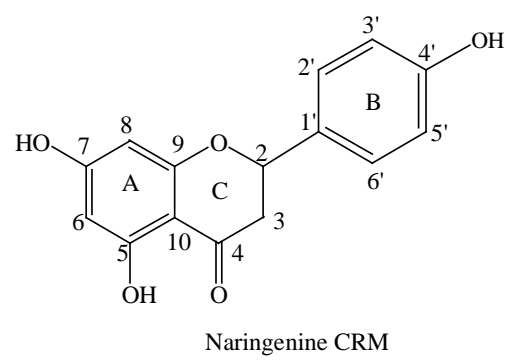

Figure 1. Structure of the studied flavonoids CRMs.

Thermo Nicolet IS10 spectrophotometers. All the flavonoids reference material solutions, diluted to concentration of $10 \mu \mathrm{g} \cdot \mathrm{mL}^{-1}$ in methanol for LC/MS Analysis were quantified at flow injection analysis mode with an Agilent 6410 triple quad system with electro-spray ionization source (ESI). The QNMR measurements were performed with a Bruker AV600 spectrometer (Bruker, Billerica, MA). The Agilent 7500CE ICP-MS was used for the analysis of trace metal residues in all the samples. A Mettler Tolerdo DL39 Karl Fischer titrator was used for water measurement of all the samples.

\subsection{Qualitative Analysis Methods}

\subsubsection{LC-MS Method}

The flavonoids CRMs in methanol with concentration of $10 \mu \mathrm{g} \cdot \mathrm{mL}^{-1}$ were used to LC-MS analysis. The ion spray voltage was set at $4.0 \mathrm{kV}$. Drying gas temperature was maintained at $350^{\circ} \mathrm{C}$ with flow rate of $9.0 \mathrm{~mL} \cdot \mathrm{min}^{-1}$. Nebulizer pressure was set at $40 \mathrm{psi}$. The fragmentor energy was set at $135 \mathrm{~V}$. The collision energy respectively were optimized at $30 \mathrm{~V}$ for baicalein and 5,7-dihydoxy flavones, and $16 \mathrm{~V}$ for naringenine. The analysis the mass spectrum peak molecular weight in an ESI positive ion mode at specified MS scan range of $60-300$.

\subsubsection{IR Method}

Infrared spectra were collected with FT-IR spectrometer Thermo Nicolet IS10 spectrophotometers via $\mathrm{KBr}$ pellets technique. Specimens were ground with dried spectroscopic grade $\mathrm{KBr}$ powder and the mixture was compressed to pellets for FT-IR measurements. The sample to $\mathrm{KBr}$ mass ratio was optimized as 1:100. All the spectra were collected in the $4000-400 \mathrm{~cm}^{-1}$ range at 4 $\mathrm{cm}^{-1}$ resolution.

\subsection{Quantitative Analysis Methods}

\subsubsection{Mass Balance Approach}

The flavonoids were quantified by HPLC equipped with an ZORBAX Eclipse XDB- $\mathrm{C}_{18}(460 \mathrm{~mm} \times 250 \mathrm{~mm}$ $\times 5 \mu \mathrm{m}$, Agilent ) column with VWD detector. The chromatography conditions are detailed in Table 1 . The purity values of flavonoids CRMs were calculated using the peak area normalization method. Following subtract the moisture and inorganic elements impurities which not measured by HPLC from $100 \%$, as follows [6,7]:

$$
\text { Purity }=\left(1-\mathrm{P}_{\mathrm{m}}-\mathrm{P}_{\mathrm{a}}\right) * \mathrm{P}_{\text {HPLC }}
$$

Where, $\mathrm{P}_{\text {HPLC }}$ is the percentage area of main compound acquired by HPLC-VWD, $\mathrm{P}_{\mathrm{m}}$ is the moisture content measured by Karl Fisher method, and $\mathrm{P}_{\mathrm{a}}$ is the inorganic elements content measured by ICP-MS.

\subsubsection{QNMR Method}

The QNMR method is based on the directly proportional relationship between the signal response (integrated signal area, $I_{x}$ ) and the number of nuclei generating the corresponding resonance line, which is represented by Eq.1 as [8].

$$
P_{x}=\frac{m_{x}}{m_{S}}=\frac{I_{x}}{I_{s t d}} \cdot \frac{N_{s t d}}{N_{x}} \cdot \frac{M_{\chi}}{M_{s t d}} \cdot \frac{m_{s t d}}{m_{S}} \cdot P_{s t d}
$$

Where $P_{x}$ is purity of analyte; $I_{x}$ and $I_{\text {std }}$ are the integrated signal areas of analyte and internal standard, respectively; $N_{x}$ and $N_{\text {std }}$ are the spin numbers of the analyte and internal standard, respectively; $M_{x}$ and $M_{\text {std }}$ are molar mass of analyte and internal standard, respectively; $m$ and $m_{\text {std }}$ are the mass of analyte and internal standard, respectively; $P_{\text {std }}$ is the purity of the internal standard.

The QNMR measurements were performed with a Bruker AV600 spectrometer (Bruker, Billerica, MA). Spectra were run with the following optimized parameters: probe size, $5 \mathrm{~mm}$; probe temperature, was $23.0^{\circ} \mathrm{C}$; excitation pulse angle, $45^{\circ}$; $32 \mathrm{~K}$ time domain points; $16 \mathrm{~K}$ spectral data points; pulse delay, $4.15 \mu \mathrm{s}$; relaxation delay, $32 \mathrm{~s}$ and number of scans, 32. A sample solution containing about $10 \mathrm{mg}$ benzoic acid, $70 \mathrm{mg}$ of carbohydrate samples diluted in $0.5 \mathrm{~mL}$ DMSO was analyzed by QNMR. The total mass of analyte in the sample solution was determined using Eq.1.

\subsection{Homogeneity Study and Stability Study}

According to the Technical Norm of Primary Reference Material of China [9], the homogeneity study and the stability study were carried out by HPLC-VWD method. 15 bottles, were randomly selected and assayed for 
Table 1. The chromatography conditions of the studied flavonoids CRMs.

\begin{tabular}{cccccc}
\hline CRMs & $\begin{array}{c}\text { Mobile phase } \\
\text { (Acetonitrile:0.1\% phosphoric acid) }\end{array}$ & Wavelength (nm) & Injection volume $(\mu \mathrm{L})$ & Flow $(\mathrm{mL} / \mathrm{min})$ & Concentration $(\mathrm{mg} / \mathrm{mL})$ \\
\hline Baicalein & $35: 65$ & 275 & 5 & 1 & 0.5 \\
5,7-dihydoxy flavones & $43: 57$ & 268 & 5 & 1 & 0.3 \\
Naringenine & $35: 65$ & 288 & 2 & 1 & 1 \\
\hline
\end{tabular}

homogeneity between bottles, and 7 portions from each bottle were assayed for homogeneity within bottle. The results were examined via $F$-test and $t$-test. In $F$-test, standard deviations were used to examine whether the deviation between bottles was significantly greater than the deviation within bottle. In $t$-test, means were used to examine whether the mean between bottles was significantly different from that of within bottle [10].

A stability study monitored a significant change in value of the flavonoids CRMs was performed over one year.

A study of long-term stability for storage at $4^{\circ} \mathrm{C}$ was carried out by HPLC-VWD method [11] in which material was determined at 1, 3, 6 and 12 months. For each condition, two solutions were prepared and analyzed in duplicate. A comparison was made between the purity determined by HPLC-VWD for different storage time. A freshly prepared control sample was analyzed at each time. All the samples were randomized for analysis.

\subsection{Uncertainty Evaluation}

The uncertainty evaluation for the flavonoids CRMs derived from the certified value, homogeneity and stability study. The expanded uncertainty was calculated as follows:

$$
U=k \cdot U_{\mathrm{c}}=k \cdot \sqrt{\mathrm{u}_{\mathrm{v}}^{2}+\mathrm{u}_{\mathrm{h}}^{2}+\mathrm{u}_{\mathrm{s}}^{2}}
$$

where, $k$ is the coverage factor, $U_{c}$ is the combined uncertainty of the sample, $u_{v}, u_{h}, u_{s}$ are the uncertainty components of the assigned value, homogeneity and stability study.

\section{RESULTS AND DISCUSSION}

\subsection{Qualitative Analysis Methods}

\subsubsection{LC-MS Method}

The experiment obtained the molecular weight of the flavonoids CRMs by full scan mass spectra. The results indicted the molecular ion peak $(\mathrm{M}+\mathrm{H})^{+}$of mass charge ratio $\mathrm{m} / \mathrm{z}$ of baicalein and 5,7-dihydoxy flavones was the molecular weight plus the atomic weight of hydrogen (1.0), the molecular ion peak $(\mathrm{M}-\mathrm{H})^{-}$of mass charge ratio $\mathrm{m} / \mathrm{z}$ of naringenine was the molecular weight subtract the atomic weight of hydrogen (1.0). Ion fragments by product ion scan are detailed in Figure 2. The results showed the flavonoids mainly fractured in 1,2 C-Ochem- ical bond and 3,4 C-C chemical bond.

\subsubsection{IR Method}

The Infrared spectrum of the flavonoids CRMs were shown in Figure 3. The results revealed the peaks at 3090, 3097, and $3114 \mathrm{~cm}^{-1}$ belong to hydroxide peaks, 1660,1653 , and $1630 \mathrm{~cm}^{-1}$ belong to associated carbonyl peaks, $1449 \sim 1618 \mathrm{~cm}^{-1}$ belong to benzene ring skeleton of $\mathrm{C}=\mathrm{C}$ peaks of the flavonoids CRMs. The Infrared spectrums of the flavonoids CRMs were consistent with those spectrums from literature [13-15] and can be used for quantification.

\subsection{Quantitative Analysis Methods}

\subsubsection{Mass Balance Approach}

The HPLC chromatogram of the flavonoids CRMs were shown in Figure 4. The results of the moisture content and the inorganic elements contents were listed in Table 2, as indicated, The water content for Baicalein, 5,7-dihydoxy flavones, and naringenine were $0.538 \%$, $0.150 \%, 0.098 \%$ and $0.115 \%$, respectively, all were less than $0.1 \%$, while the inorganic content all warless than $0.00002 \%$ at the studied conditions.

\subsubsection{QNMR Method}

QNMR was also used to determinate purity values flavonoids CRMs. The NMR spectrums were shown in Figure 5. The $\mathrm{C}_{3}-\mathrm{H}$ peak areas of baicalein, $\mathrm{C}_{8}-\mathrm{H}$ peak areas of 5,7-dihydoxy flavones, $\mathrm{C}_{4},-\mathrm{OH}$ peak areas of naringenine, $\mathrm{C}_{2,2}-2 \mathrm{H}$ peak areas of the benzoic acid internal standard were selected for integration.

\subsubsection{Purity Values of the Flavonoids CRMs}

The purity values of flavonoids CRMs were calculated with the average of mass balance approach and QNMR method. The results are listed in Table 2.

\subsection{Homogeneity Study and Stability Study}

The $F$-Test and $t$-test results of all the studied were summarized in Table 3. No significant differences were found for all the studied compounds in homogeneity test as the calculated $F$-values were less than the critical $F$-values (Table 3). Although a slight difference was observed, this was attributed to the variation of the analytical method. 


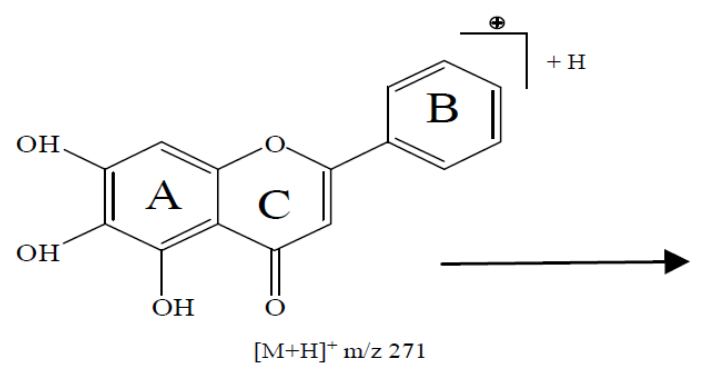

Baicalein CRM<smiles>O=C1C=C(O)C(O)=C(O)C1=C=[OH+]</smiles>

$\mathrm{m} / \mathrm{z} 169$

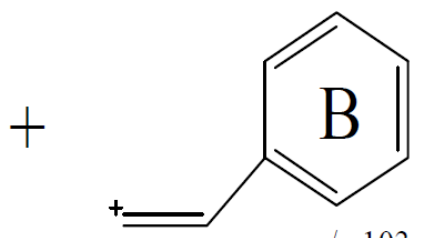

$\mathrm{m} / \mathrm{z} 103$<smiles>C=Cc1ccccc1</smiles>

5,7-dihydoxy flavones CRM

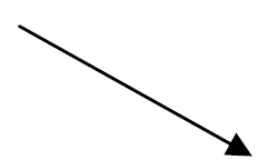<smiles></smiles>

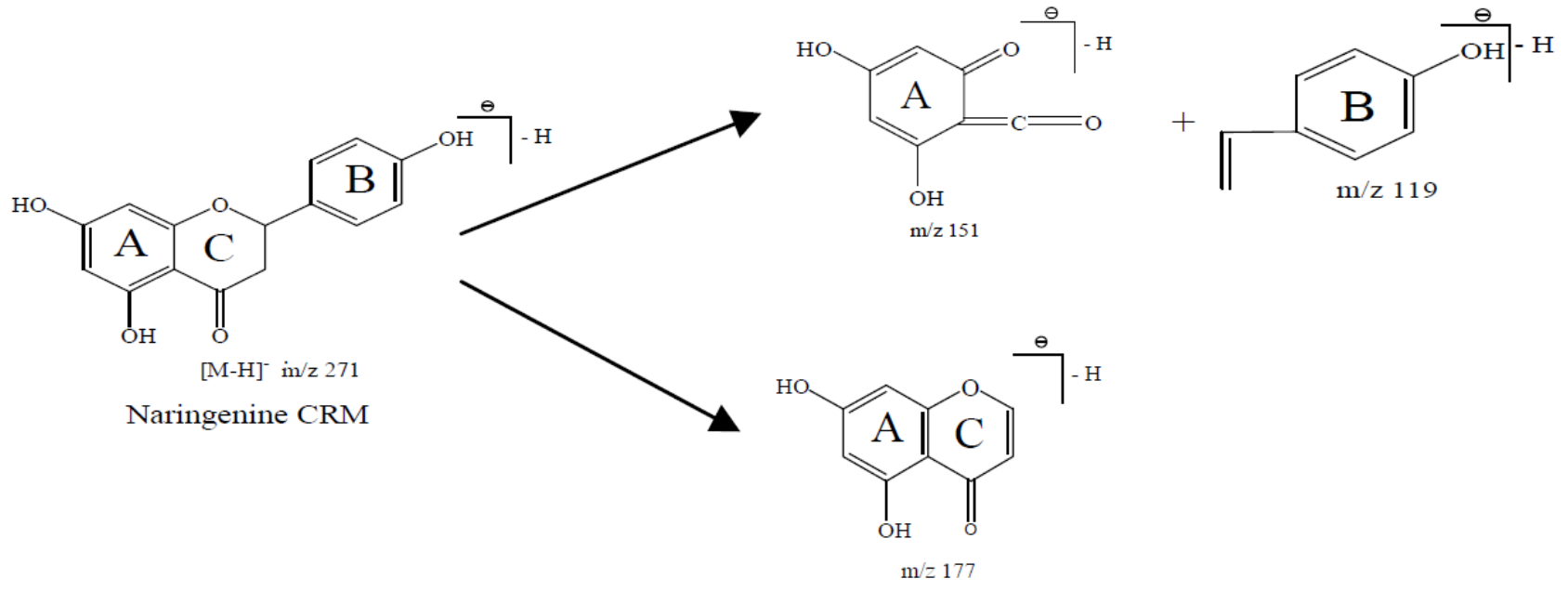

Figure 2. Main fragmentation schemes of the flavonoids CRMs.
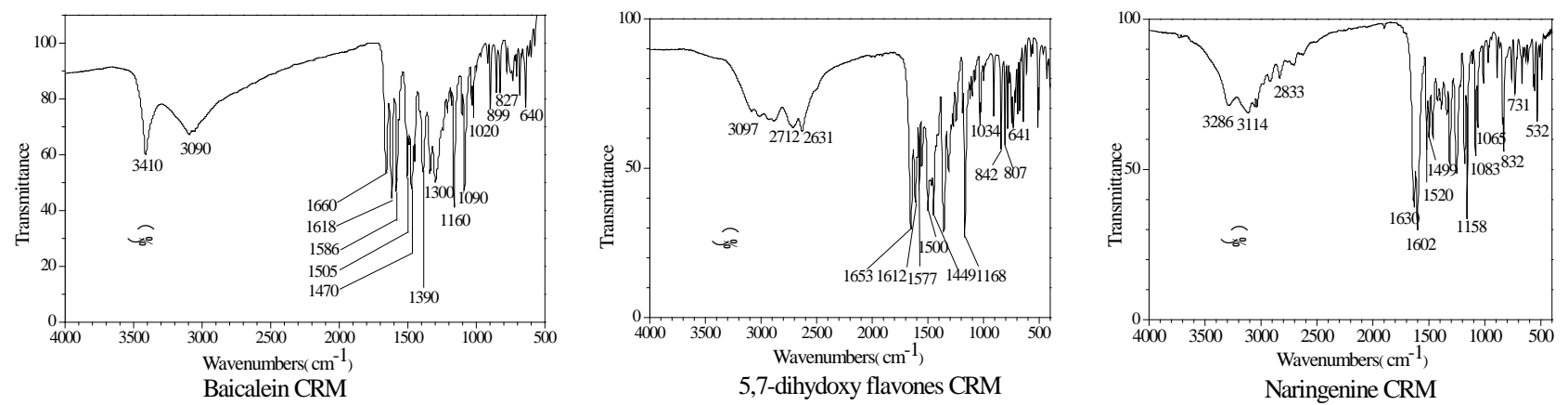

Figure 3. The infrared spectrums of the studied flavonoids CRMs.

The flavonoids CRMs stored at $4^{\circ} \mathrm{C}$ were used to test stability. The testing data of stability study are showed in
Table 4. The results in different times were consistent with the standard value through the $t$-test analysis. There 


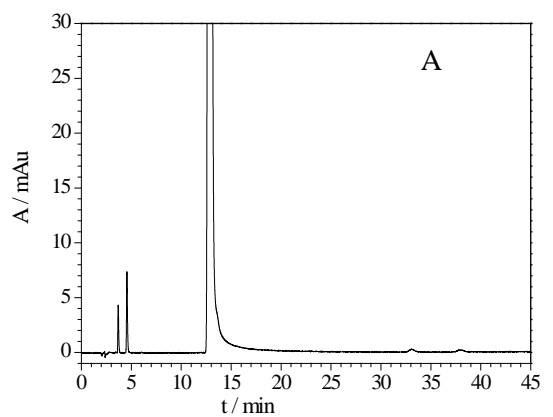

Baicalein CRM

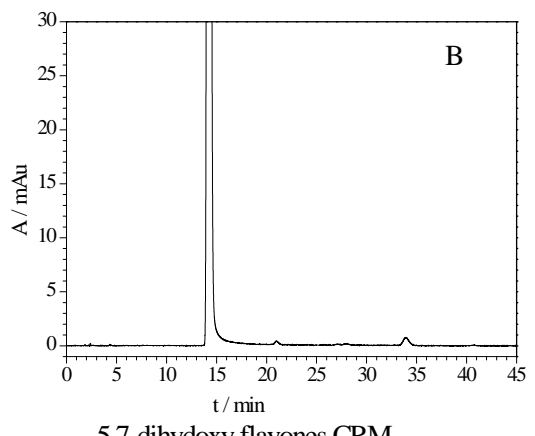

5,7-dihydoxy flavones CRM

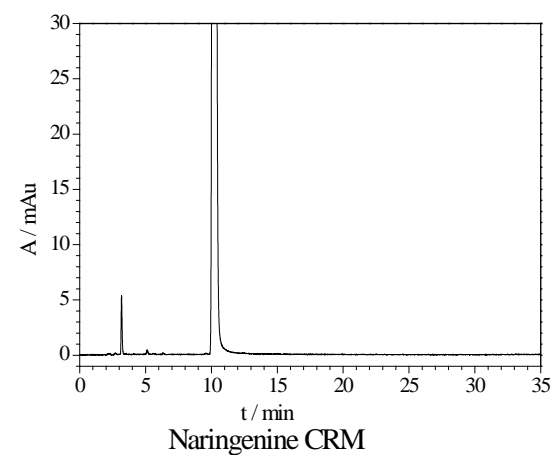

Figure 4. Purity determination HPLC chromatogram of the flavonoids CRMs.

Table 2. The purity values results of flavonoids CRMs.

\begin{tabular}{cccc}
\hline CRMs & HPLC (\%) & QNMR (\%) & Average (\%) \\
\hline Baicalein & 98.78 & 98.88 & 98.8 \\
5,7-dihydoxy flavones & 99.40 & 98.84 & 99.1 \\
Naringenine & 99.47 & 99.46 & 99.5 \\
\hline
\end{tabular}

were no obvious changes in purity values monitored of the flavonoids CRMs over one year. The results mean that they were stable.

\subsection{Uncertainty Evaluation}

The uncertainty for the flavonoids CRMs is composed of the uncertainty from the certified value, homogeneity and stability study.

\subsubsection{Uncertainty of Certified Value}

1) Uncertainty from HPLC measurement [16].

a) $u_{1}$ (type A uncertainty) is the uncertainty from repeatability of HPLC measurement.

b) $u_{2}$ (type B uncertainty) is the uncertainty from different responses from flavonoids CRMs and impurities at different wavelengths of HPLC measurement.

The combined uncertainty is as follow:

$$
\mathrm{u}_{\mathrm{HPLC}}=\sqrt{u_{1}^{2}+u_{2}^{2}}
$$

2) Uncertainty of the QNMR measurement

a) $u_{3}$ (type A uncertainty) is the uncertainty from repeatability of QNMR measurement.

b) $u_{4}$ is the uncertainty from relative molar masses of flavonoids CRMs and benzoic acid internal standard CRM. The value of $u_{4}$ calculated is less than $0.1 \%$. In this case, $\mathrm{u}_{4}$ is ignored.

c) $u_{5}$ is the uncertainty from benzoic acid CRM. The uncertainty of benzoic acid CRM was $0.1 \%$, according to the uniform distribution, $\mathrm{u}_{5}=0.1 \% / 3^{1 / 2}=0.06 \%$.

d) $u_{6}$ is the uncertainty from balance weighing. The balance (Max $=2.1 \mathrm{~g}, \mathrm{~d}=0.1 \mu \mathrm{g}$ ) was used to weigh the flavonoids CRMs and benzoic acid internal standard CRM. Balance changing and test error estimate for the $0.5 \mu \mathrm{g}$, therefore $\mathrm{u}_{6}$ is estimated $0.01 \%$.
The combined uncertainty is as follow:

$$
\mathrm{u}_{\mathrm{QNMR}}=\sqrt{\mathrm{u}_{3}^{2}+\mathrm{u}_{4}^{2}+\mathrm{u}_{5}^{2}+\mathrm{u}_{6}^{2}}
$$

\subsubsection{The Combined Uncertainty}

The combined uncertainty for the flavonoids CRMs is composed of the uncertainty from the certified value, homogeneity and stability study. The results of homogeneity and stability study completely conformed to the requirement of homogeneity and stability in the regulation of the CRMs. The homogeneity and stability of the flavonoids CRMs both are good. Therefore the uncertainty of homogeneity $\left(\mathrm{u}_{h}\right)$ and stability $\left(\mathrm{u}_{\mathrm{s}}\right)$ is neglected.

$$
\begin{aligned}
\mathrm{U}_{\mathrm{C}} & =\sqrt{\mathrm{u}_{\mathrm{v}}{ }^{2}+\mathrm{u}_{\mathrm{h}}^{2}+\mathrm{u}_{\mathrm{s}}^{2}}=\sqrt{\mathrm{u}_{\mathrm{HPLC}}{ }^{2}+\mathrm{u}_{\mathrm{QNMR}}^{2}+\mathrm{u}_{\mathrm{h}}^{2}+\mathrm{u}_{\mathrm{s}}{ }^{2}} \\
& =\sqrt{\mathrm{u}_{1}{ }^{2}+\mathrm{u}_{2}^{2}+\mathrm{u}_{3}{ }^{2}+\mathrm{u}_{4}^{2}+\mathrm{u}_{5}{ }^{2}+\mathrm{u}_{6}^{2}+\mathrm{u}_{\mathrm{h}}^{2}+\mathrm{u}_{\mathrm{s}}^{2}} \\
& \approx \sqrt{\mathrm{u}_{1}{ }^{2}+\mathrm{u}_{2}^{2}+\mathrm{u}_{3}{ }^{2}+\mathrm{u}_{4}^{2}+\mathrm{u}_{5}{ }^{2}+\mathrm{u}_{6}^{2}}
\end{aligned}
$$

\subsubsection{The Expanded Uncertainty}

The expanded uncertainty $(U)$ for this flavonoids CRMs was calculated by multiplying the combined uncertainty by a coverage factor of $k=2$. The uncertainty evaluation for flavonoids CRMs were listed in Table 5.

\section{CONCLUSION}

The three flavonoids CRMs were developed with the procedures of sample preparation, certified value, homogeneity study, and stability study and uncertainty evaluation. The three flavonoids CRMs can not only meet the requirement of quantitative detection of food, medicine and related materials, but also provide a more quickly and accurately experimental methods for the research and development of related products.

\section{ACKNOWLEDGEMENTS}

The authors acknowledge Special Program for Key Basic Research of the Ministry of Science and Technology, China (Grant No. 2011FY130100) and the National Natural Science Foundation of China (Grant No 21275134/B0505) for financing this work. 

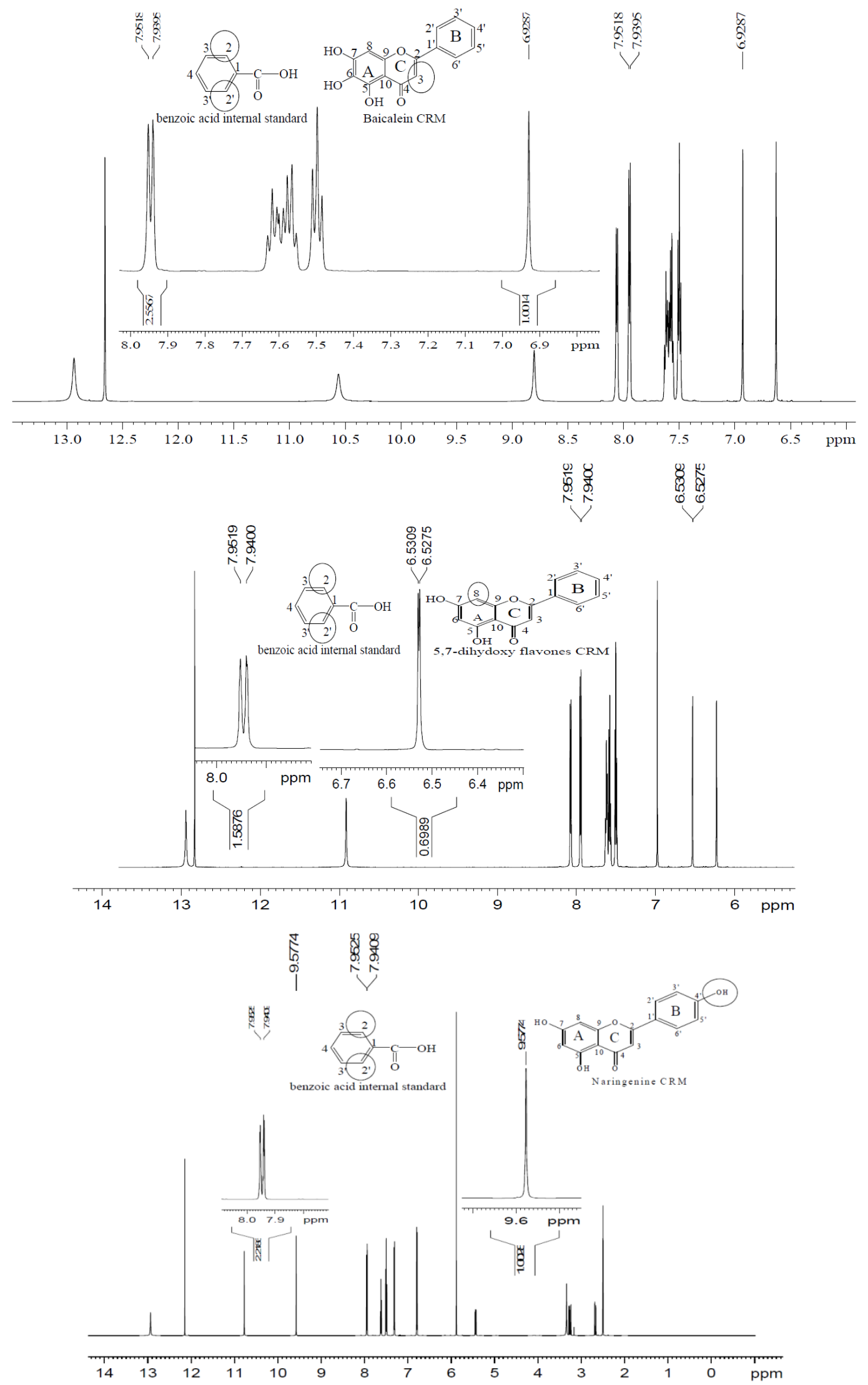

Figure 5. The spectrums of the studied flavonoids CRMs. 
Table 3. Homogeneity results for the contents, the within-bottle, and between-bottle and homogeneity test of flavonoids CRMs.

\begin{tabular}{|c|c|c|c|c|}
\hline \multicolumn{2}{|c|}{ Homogeneity test } & Baicalein CRM & 5,7-dihydoxy flavones CRM & Naringenine CRM \\
\hline \multirow{2}{*}{$\bar{X}$} & between-bottle $\left(\mathrm{s}_{1}, \%\right)$ & $99.31 \pm 0.018$ & $99.58 \pm 0.013$ & $99.58 \pm 0.015$ \\
\hline & within-bottle $\left(\mathrm{s}_{2}, \%\right)$ & $99.31 \pm 0.011$ & $99.58 \pm 0.010$ & $99.58 \pm 0.010$ \\
\hline \multirow{3}{*}{$F$-test } & $F=s_{1}^{2} / s_{2}^{2}$ & 2.63 & 1.86 & 2.60 \\
\hline & $\mathrm{F}_{0.05}(14,6)$ & 3.96 & 3.96 & 3.96 \\
\hline & $\mathrm{F}<\mathrm{F}_{0.05}$ & $\mathrm{Y}$ & $\mathrm{Y}$ & $\mathrm{Y}$ \\
\hline \multirow{3}{*}{$t$-test } & $\mathrm{t}=\left|\bar{X}_{1}-\bar{X}_{2}\right|$ & $0.0010 \%$ & $0.0011 \%$ & $0.0022 \%$ \\
\hline & $\mathrm{t}_{a}=2 \mid\left(\frac{\mathrm{s}_{1}}{\sqrt{\mathrm{n}_{1}}}\right)^{2}+\left(\frac{\mathrm{s}_{2}}{\sqrt{\mathrm{n}_{2}}}\right)^{2}$ & $0.0064 \%$ & $0.0049 \%$ & $0.0053 \%$ \\
\hline & $\mathrm{t} \leq \mathrm{t}_{\alpha}$ & $\mathrm{Y}$ & $\mathrm{Y}$ & $\mathrm{Y}$ \\
\hline
\end{tabular}

Table 4. The results of stability of flavonoids CRMs.

\begin{tabular}{cccc}
\hline Time interval & Baicalein & 5,7-dihydoxy flavones & Naringenine \\
\hline Zero month & 99.27 & 99.50 & 99.53 \\
One month & 99.37 & 99.56 & 99.57 \\
Three months & 99.31 & 99.54 & 99.58 \\
Six months & 99.31 & 99.52 & 99.50 \\
Twelve months & 99.35 & 99.46 & 99.55 \\
Average (\%) & 99.32 & 99.52 & 99.55 \\
RSD (\%) & 0.04 & 0.04 & 0.03 \\
\hline
\end{tabular}

Table 5. The results of uncertainty evaluation of flavonoids CRMs.

\begin{tabular}{|c|c|c|c|}
\hline Uncertainty & Baicalein CRM & 5,7-dihydoxy flavones CRM & Naringenine CRM \\
\hline$u_{1} / \%$ & 0.01 & 0.02 & 0.03 \\
\hline$u_{2} / \%$ & 0.04 & 0.04 & 0.01 \\
\hline$u_{3} / \%$ & 0.36 & 0.33 & 0.50 \\
\hline$u_{4} / \%$ & 0 & 0 & 0 \\
\hline$u_{5} / \%$ & 0.06 & 0.06 & 0.06 \\
\hline$u_{c} / \%$ & 0.37 & 0.34 & 0.50 \\
\hline $\mathrm{k}$ & 2 & 2 & 2 \\
\hline$U / \%$ & 0.8 & 0.7 & 1.0 \\
\hline Purity value/\% & 98.8 & 99.1 & 99.5 \\
\hline
\end{tabular}

\section{REFERENCES}

[1] Arunachalama, J., Bleiseb, A., Mahwar, R.S., et al. (2006) The natural matrix reference material database of the International Atomic Energy Agency: Reference materials in support of dietary investigations. Journal of Food Composition and Analysis, 19, 241-249. http://dx.doi.org/10.1016/j.jfca.2005.07.002

[2] Sander, L.C., Sharpless, K.E. and Wise, S.A. (2006) Dietary supplement standard reference materials. Life Sciences, 78, 2044-2048.

http://dx.doi.org/10.1016/j.lfs.2005.12.037

[3] Chen, C.H., Huang, T.S., Wong, C.H., et al. (2009) Synergistic anti-cancer effect of baicalein and silymarin on human hepatoma HepG2 Cells. Food and Chemical Toxicology, 47, 638-644. http://dx.doi.org/10.1016/j.fct.2008.12.024

[4] Middleton Jr., E. and Kandaswami, C. (1992) Effects of flavonoids on immune and inflammatory cell functions. Biochemical Pharmacology, 43, 1167-1179. http://dx.doi.org/10.1016/0006-2952(92)90489-6

[5] Heim, K.E., Tagliaferro, A.R. and Bobilya, D.J. (2002) Flavonoid antioxidants: Chemistry, metabolism and structure-activity relationships. Journal of Nutritional Biochemistry, 13, 572-584.

http://dx.doi.org/10.1016/S0955-2863(02)00208-5

[6] The Directorate for the Quality of Medicines of the Council of Europe (2007) Establishment of reference standards, in European Pharmacopoeia. 5th Edition, Supplement 5.6, General texts 5.12.4, Council of Europe, Strasbourg.

[7] World Health Organization (2003) Evaluation of chemi- 
cal reference substance. In: International Pharmacopoeia, 3rd Editon, Vol. 5, Supplementary Information, Part A, 3.3, World Health Organization, Geneva.

[8] Liu, S.Y. and Hu, C.Q. (2007) A comparative uncertainty study of the calibration of macrolide antibiotic reference standards using quantitative nuclear magnetic resonance and mass balance methods. Analytica Chimica Acta, 602, 114-121. http://dx.doi.org/10.1016/j.aca.2007.09.009

[9] JJG1006-1994 (1994) Technical norm of primary reference material of china. Beijing.

[10] Quan, H. (2002) Reference material and its application technology. China Standard Press, Beijing. (in Chinese)

[11] Dai, X.H., Fang, X., Shao, M.W., Li, M., et al. (2011) Certified reference materials (GBW09170 and 09171) of creatinine in human serum. Journal of Chromatography B, 879, 429-435.

http://dx.doi.org/10.1016/j.jchromb.2010.12.031
[12] EURACHEM/CITAC Guide CG4 (2000) Quantifying uncertainty in analytical measurement. Seconded.

[13] Li, Y.X., Suo, Q.L., He, W.Z., et al. (2008) Separation and Purification and Structural Characterization of Baicalein. Spectroscopy and Spectral Analysis, 28, 18951899.

[14] NIST, NIST Chemistry WebBook. http://webbook.nist.gov/

[15] Wang, Y.G., Xie, S.W. and Su, W.W. (2005) Study on the chemical constituents of Polygonum chinese L. Journal of Chinese Medicinal Materials, 28, 1000-1001.

[16] Quan, C., Su, F.H., Wang, H.F., et al. (2011) Development of anabolic-androgenic steroids purity certified reference materials for anti-doping. Steroids, 76, 1527-1534. http://dx.doi.org/10.1016/j.steroids.2011.09.001 